\title{
Cross-border Marketing Mode for Commercial Banks in Mobile Internet Era
}

\author{
Xuehong Zhao \\ School of Management \\ Harbin Finance University \\ Harbin, 150030, China
}

\begin{abstract}
The mobile Internet era breaks the original closed business model and changes people's way of life and consumption. Cross-border marketing can open up new living space for enterprises or brands, and also can make brands glow with new vitality due to superimposed synergies. Taking commercial banks as the research object, this work discussed how commercial banks conduct cross-border marketing. Based on the necessity of cross-border marketing by commercial banks, the principle, which commercial banks should grasp cross-border marketing, was clarified. Combined with the characteristics of commercial banks, how to carry out cross-border marketing was clarified into four aspects: product cross-border, channel crossborder, promotion cross-border, and cultural cross-border.
\end{abstract}

Keywords-Mobile Internet Era; Commercial Banks; CrossBorder Marketing

\section{INTRODUCTION}

Cross-border marketing is a new marketing mode which breaks the traditional marketing mode, referring to cooperation between brands in different industries. The novelty and interesting performance generated by cross-border marketing can attract people's attention. Its greatest benefit is to let the original irrelevant elements infiltrate and integrate with each other, meet the needs of users from various angles, make users stick to the brand and enrich it, and give the brand a sense of three-dimensional and depth [1]. Marketing boundaries, breaking through the tradition, is a sublimation of traditional marketing concepts and models.

China's Internet development is focused on traffic economy. No matter what a product actually is, as long as it can bring super-high traffic, it will be able to achieve big sales. Although some are blind, some enterprises can realize the empowerment of other industries through such cross-border integration. Since 2018, more and more brands have played cross-border marketing. Maxam and White Rabbit have joined forces to launch the White Rabbit Toffee Lip Balm. Musk Hemorrhoids Ointment and 999 Dermatitis Flat both introduced lipsticks, and 999 Dermatitis Flat were named as "Heding Red", "Sunset Red" and "Enough Orange", respectively. Cross-border marketing combines and extends some elements that are not related to each other according to

This research was funded and supported by the following items:

1)Fundamental scientific research project of undergraduate universities in Heilongjiang Province in 2017 (2017-KYYWF-0083).

2)Research planning project on philosophical and social sciences in Heilongjiang Province in 2018 (18JLE519).

3)Fundamental scientific research project of undergraduate universities in Heilongjiang Province in 2019 (2019-KYYWF-010). the commonness and connection among consumers of different industries, products and preferences. It highlights a unique attitude towards life, aesthetic taste or values to win the favor of target consumers, so as to realize the new marketing mode of market maximization and profit maximization in crossborder joint ventures [2].

\section{ThE NeCESSITY OF CROSS-Border MARKETING FOR COMMERCIAL BANKS}

The essence of cross-border marketing is to realize that multiple brands interpret the same user characteristics from different perspectives. The products of commercial banks include not only tangible products, but also intangible services. In the past, the advantages of enterprises in commodity and technology have been weakened by the characteristics of Internet opening and sharing. The market competition is increasingly fierce, accompanied by the development obstacles of commodity homogenization, market behavior imitation and disorderly competition. Therefore, the competition focus of enterprises is gradually changed from pure commodity and service innovation to competing for consumers. Cross-border marketing poses many challenges to mutually cooperative companies in terms of marketing capabilities.

Cross-border marketing can aggregate dispersed and effective factors into complete and better resources. The new consumer groups represented by the post-80s and post-90s have brand-new consumption habits and characteristics, and their super consumption ability promotes the development of cross-border marketing. The Internet has broken the original development pattern. The products launched by enterprises need to be updated iteratively and recombined. In this process, cross-border has become more common and diversified. KFC not only sells fried chicken, but also has nail polish and sunscreen, and Coca-Cola has made perfume brand. Crossborder cooperation and brands no longer fight alone. Commercial banks can offer more value-added services for customers, strengthen the actual interaction with customers, effectively convey ideas and services, and enhance business capabilities and service levels [3].

\section{The PrinCIPLE OF CROSS-Border MARKETING FOR COMMERCIAL BANKS}

Cross-border marketing seeks external resources similar to the target customer group or brand for cooperation through horizontal analysis of target customer groups or target market 
products. in order to meet customers' complex and diverse life pursuits and generate new competitive advantages. When implementing cross-border marketing strategy, commercial banks should grasp the following principles:

\section{A. Resource matching principle}

The key step to develop cross-border marketing is to do a good matching job. Matching high-quality partners and ways of cooperation can help cross-border marketing achieve twice the result with half the effort. Both brands have certain brand value in their own fields. Only when they work together can they be helpful to enhance the brand value in both sides. In cross-border marketing, two different brands of enterprises should have commonality and equivalence in enterprise strategy, marketing ability, consumer groups, market position, etc., so as to better play the synergistic effect.

\section{B. Mutual benefit principle}

Cross-border marketing is not joint promotion. At least peers can not stand together to shout. The purpose of crossborder marketing is to enrich the connotations for their products or brands through cooperation, to achieve the promotion of both sides in brand or product sales (i.e., the enterprises or brands participating in cross-border marketing should be mutually beneficial and mutually reinforcing symbiotic relationships rather than the competitive relationship of the competition. Enterprises participating in cooperation should not be competitive in brand. Through friendly consultation and full cooperation, both sides can achieve the best results in cross-border marketing and find an interest balance between both sides [4].)

\section{User experience principle}

Cross-border marketing should focus on the establishment of complementary relationship for user experience. The basis for finding partners is the complementary for user experience and not simple functional complementarity. In cross-border cooperation, each party extracts the core elements according to its own advantages and characteristics, seeks the fit point with the other party's core elements, and improves the common user experience from multiple dimensions. They penetrate and integrate with each other, thus giving the joint brand a sense of three-dimensional and depth, which can impress users and help customers solve problems. The user feels sincerity and friendliness, reflecting professionalism and arousing the user's resonance [5].

\section{THE WAY OF CROSS-BORDER MARKETING FOR COMMERCIAL BANKS}

\section{A. Product cross-border}

Product cross-border can let it bear more additional attributes. Commercial banks should focus on how to use AI to promote marketing upgrade and achieve precision marketing in mobile Internet era. Sogou and SPD Bank credit card jointly created "Everything is IN Your AI--SPD Bank Credit Card * Sogou Cross-border Marketing" which won many awards. The background design of "Wangzai", which best represents the strength of Sogou AI technology, was integrated into it. The perfect integration of Sogou AI attribute and SPD Bank credit card financial attribute was realized, and the brand image of SPD Bank credit card actively embracing AI and financial technology are successfully shaped.

Air travel customers have high consumption capacity, but also have strong demand for wealth management. Based on the travel wealth card, CGB and airlines cross-border hand in hand to provide customers with a comprehensive package of services covering finance and navigation. CGB integrates superior resources and high-end services, aiming at airline passengers who are highly compatible with its wealth customers, and launches a wealth debit card that integrates financial and travel services. Free air tickets can also be obtained in bank financing, and mileage can be exchanged through credit card consumption. The travel wealth card launched by CGB is a pioneer in the industry, which will offer customers with more choices by converting the scale of wealth into air mileage. Through crossborder cooperation and resource integration, the advantages of financial + non-financial integrated services created by commercial banks can effectively enhance customer stickiness, further expand service groups and promote the transformation and development of commercial banks [6].

\section{B. Channel cross-border}

Channel cross-border means that two cooperative brands cooperate on the basis of channel sharing to obtain consumers in different fields. The existing conventional channels are constantly expanding and developing to new mobile Internet channels in mobile Internet era. Off-line channels are constantly merging with on-line channels to create a threedimensional and omni-directional channel system. Based on the product matrix covering 600 million precise users, SPD Bank's credit card "cross-border" Sogou takes advantage of intelligent technology support; target group refined operation and diversified functional layout, etc. Sogou opens many big traffic ports (e.g., Sogou input method, Sogou website navigation, Sogou financing channel) to expand online channels for SPD Bank credit cards and to drain new users. High-quality traffic for SPD Bank credit cards are gathered.

Consumption scenarios and user channels become key factors in mobile Internet era. Commercial banks can offer consumer financial services through credit cards and consumer credit, while consumer financial companies focus on customizing processes around consumer needs and making customer experience faster and more convenient. Cross-border channels can deepen the integration and penetration with each other, and enhance the support and cooperation between enterprises. In the process of creating the whole channel pattern, the market layout of enterprises will be more three-dimensional and systematic. CMB online banking and NetEase recharge system cross-border cooperation "CMB online banking recharge lucky draw". This is the first time that NetEase has developed a program for brand customers and built a consumption platform for the target population of CMB online banking. Based on the NetEase game recharge center, this promotion selects the recharge center to over 10 million stations to promote the resources, expand the exposure coverage, bring the recharge center advertising impressions beyond 300 million, and encourage the CMB brand to continue 
to emerge. This is also the embodiment of the resource matching principle.

Commercial banks have a large amount of customer cash flow, bank card holders and user financial data, which are not available in traditional e-commerce. However, when using ecommerce channels, problems such as poor shopping experience and after-sale services should be avoided. When choosing cooperative e-commerce, It is necessary to make up for the weakness to commercial banks in this respect in terms of not only the technical ability and data mining ability based on mobile Internet, but also marketing and user experience [7].

\section{Promotion cross-border}

Promotion cross-border refers to the cooperative intention of a cooperative enterprise to achieve promotion. One party regards the other party's products as promotional products or promotional tools of its own products or the products of both parties are promotional products for each other. As a marketing medium, express bags have their unique advantages. Advertising on express bags is a marketing scene with high attention and good feeling for users. CMB credit card and Amazon's express bags have become "web celebrity" with "my wish is to buy the world full of love", "one day's plan is to brush, one year's plan is to brush Fun", etc., arousing the resonance of the audience.

Sogou cooperated with SPD Bank credit card to create the industry-wide event of "2008 Sogou in Panoramic Election Ceremony", launch online votes, and customize thematic promotions. The launch of Sogou product matrix attracted a large number of netizens to participate, and truly realized the brand's massive exposure and marketing transformation. SPD Bank, in conjunction with the famous cartoonist "Uncle $\mathrm{Hu}$ Yin", has created a number of posters in nostalgic style around its "intentional" channel, and replaced them with application scenarios. Combined with the four great classical characters, it shows the function and selling point of "intentionality": conveying the mind and making the impossibility possible. China CITIC Bank red equity brand created by China CITIC Bank makes high-profile Big Day for users and presents Magic Show for the first time in financial circles. It not only brings magic skills into full play, but also brings in a group of friends, such as iQIYI, ofo, Little Red Book, Haagen Dazs, Hammer, King Hamburg, etc., which successfully attracted the attention of young people.

\section{Culture cross-border}

The mobile Internet era accelerates the diversification and integration of cultures. Enterprises need to cross cultural boundaries in order to achieve localization marketing or activation of old brands. They need to make use of traditional culture to create momentum for products, or take advantage of regional advantages to further enhance product connotation and promote brand value for products.

Qixi Festival is known as "Chinese Valentine's Day". With the gradual deepening of Chinese young people's understanding for traditional culture, this festival has been paid more and more attention by major brands, and has become a good opportunity to build brand awareness and reputation. On the Qixi in 2016, CMB credit card, at the busiest Tokyo Shibuya intersection in the world, where 3000 people pass every minute, has contracted five large screens. With "you know I am waiting for you", "special love to special you" and other romantic words, a new "love story" of the CMB credit card and its users has been staged, becoming the most arresting sight. Cross-border marketing should carry out interesting and valuable marketing activities from the perspective of users, and link brands, cultures and consumers to form good interaction with users. A good reputation is formed and spread out in a continuous explosion. For emotional resonance, cultural guidance is the best fuse, hitting the scene pain point of consumers.

Huishang Bank and Fuxin Media launched the "joint name card". Through the innovative development model of "culture + finance", cross-border cooperation can be realized. A new platform for financial support to cultural development can be built together, which can maximize the experience of customer card use and promote the deep integration of culture and finance.

\section{CONCLUSION}

The arrival of mobile Internet era has broken the original closed business model, and profoundly changed people's way of life and consumption. "Cross-border" has become a new normal and new theme. Cross-border marketing has subverted the traditional idea of brand, brought consumers a better user experience, and realized the sharing of resources. It is necessary to find a match between the product and the consumer's sales logic, take into account the brand's tonal innovation, and effectively reach the customer. Cross-border has become a trend, representing a new sharp attitude towards life. The cross-border road has brought more and more in-depth cooperation and sharing. Based on the users, it should make use of complementary resources to achieve win-win situation.

\section{REFERENCES}

[1] Zhuo Man. Research on Successful Elements and Implementation Path of Cross-border Marketing [J]. Journal of Changchun Normal University, 2017 (06): 196-198.

[2] Li Zhijun. Cross-border Marketing [J]. Chinese Clothing, 2019.06:72-73.

[3] He Lijun. Research on Cross-border Marketing Strategy of Agricultural Products [D]. Master's Degree Thesis, Yantai University, 2018.

[4] Qiu Li. Research on Cross-border Marketing Strategies of Industrial Bank A Products and Jiulongzhai B Products [D]. Master's Degree Thesis, Jiangxi University of Finance and Economics, 2017.

[5] Qi Yuan. Research on Marketing Strategy of Q Commercial Bank under the Background of Internet Finance [D]. Master's Degree Thesis, Yunnan Normal University, 2017.

[6] Yang Yu. Research on Cross-border Marketing in the Era of "Internet +" [D]. Master's Degree Thesis, Heilongjiang University, 2018.

[7] He Shuangshuang. Cross-border Marketing: Complementarity and Synergy "Two-Pronged Approach" [J]. Operations and Management, 2018 (10): 119-122. 